\title{
Evaluation of cross-reactivity between casein components using inhibition assay and in silico analysis
}

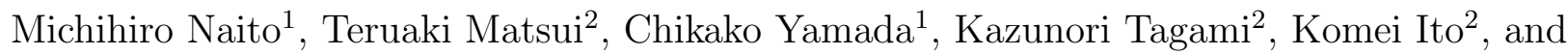 \\ Hidehiko Izumi ${ }^{1}$ \\ ${ }^{1}$ Nagoya University of Arts and Sciences \\ ${ }^{2}$ Aichi Children's Health and Medical Center
}

June 25, 2020

\begin{abstract}
Background: We previously reported that the specific IgE levels to $\alpha$ s1-casein $(\mathrm{CN})$ and $\beta$-CN in patients with cow's milk allergy decreased with similar dynamics during oral immunotherapy. Therefore, we hypothesized that $\alpha$ s- and $\beta$-CN have strong crossreactivity among $\mathrm{CN}$ components, despite the low similarity in the full-length amino acid sequences. Methods: The $\alpha$ s1-, $\beta$-, and $\chi$-CN were purified from commercial cow's milk. We recruited 39 patients with cow's milk allergy and the serum IgE levels for each CN component were measured by enzyme-linked immunosorbent assay (ELISA). Cross-reactivity between CN components was investigated by competitive ELISA against $\alpha$ s1-CN. Sequence homology between CN components at the peptide level was calculated using in silico analysis and quantified by the Property Distance (PD) value. Results: The $\alpha$ s1-CN-specific IgE levels exhibited a strong positive correlation with the $\beta$-CN-specific $\operatorname{IgE}(\mathrm{r}=0.945, \mathrm{P}<0.001)$. Complete competition was observed by $\beta$-CN against $\alpha$ s1-CN, suggesting the presence of common epitopes between them. In silico analysis detected 24 peptide sets with PD values lower than 10 between $\alpha$ s1- and $\beta-\mathrm{CN}$, and 14 sets between $\alpha$ s1- and $\chi$-CN. The amino acid sequences of $\alpha$ s1- (E61-E70) and $\beta$-CN (I12-E21) that showed the lowest PD value (5.30) were present in the characteristic sequence known as casein phosphopeptide (CPP). Conclusion: We detected strong cross-reactivity between CN components. Furthermore, we found highly homologous sequences in the CPP region, which contains a core sequence of "SSSEE" with phosphorylated serine residues.
\end{abstract}

\section{INTRODUCTION}

Casein $(\mathrm{CN})$, a major allergen for patients with persistent cow's milk (CM) allergy, comprises approximately $80 \%$ of CM proteins. ${ }^{1}$ Bovine $\mathrm{CN}$ is constituted of four components, $\alpha \mathrm{s} 1-, \alpha \mathrm{s} 2-, \beta-$, and $\chi$-CN, and exists as a micelle with a $30-300 \mathrm{~nm}$ diameter. ${ }^{2} \mathrm{CN}$ is an unfolded phosphoprotein and interacts with calcium phosphate in milk. ${ }^{3}$ as-CN is defined as an insoluble fraction in $0.4 \mathrm{M}$ calcium chloride, while $x$-CN is the soluble fraction. $\beta-\mathrm{CN}$ is defined as soluble in $3.3 \mathrm{M}$ urea and insoluble in $1.7 \mathrm{M}$ urea. Bovine $\alpha$ s1-, $\alpha$ s2-, and $\beta$-CN are comprised of 199,207 , and 209 residues, respectively, and are calcium-sensitive. ${ }^{3}$ Bovine $x$-CN is a glycoprotein comprised of 169 residues and is not calcium-sensitive. The homology of amino acid sequences among CN components is extremely low (Table S1).

We previously reported that specific IgE levels to purified $\alpha$ s1-CN and $\beta-\mathrm{CN}$, but not $\chi$-CN, showed simultaneous decreases during CM oral immunotherapy. ${ }^{4}$ Therefore, we hypothesized that as1- and $\beta$-CN have cross-reactive epitopes, or alternatively, these two CNs might share remarkably similar antigenic properties. In this study, we investigated cross-reactivity among CN components.

Furthermore, Candreva et al. analyzed the in silico similarity between proteins at the peptide level. ${ }^{5}$ Crossreactivity between milk and soy proteins has been suggested by other immunological studies. ${ }^{6}$ Thus, we 
also employed an in silico analysis at the peptide level using the peptide similarity tool on the Structural Database of Allergenic Proteins (SDAP).

\section{METHODS}

\subsection{Materials}

Each CN component ( $\alpha$ s1-, $\beta$-, and $x$-CN) was prepared following the previously described methods. ${ }^{4}$ ProteinFree Blocking Buffer (PFBB) was purchased from Thermo Fisher Scientific (Roskilde, Denmark). Alkaline phosphatase (AP)-conjugated goat anti-human IgE was purchased from Bethyl Laboratories (Montgomery, AL, USA). Anti- $\alpha$-casein mouse monoclonal antibody was purchased from Cosmo Bio (Tokyo, Japan; out of production). Peroxidase-conjugated anti-mouse IgG was purchased from Jackson ImmunoResearch (PA, USA).

\subsection{Serum samples}

This study used serum from 39 patients [median age 8 years (IQR 6-10 years)], from a previous study, with persistent CM allergy before oral immunotherapy. ${ }^{4,7}$ These patients were required to have retained their milk allergy over age 5 with the threshold dose less than $5 \mathrm{~mL}$ of $\mathrm{CM}$ confirmed by an oral food challenge test, and detectable specific IgE to $\mathrm{CM}$ and whole $\mathrm{CN}$ by ImmunoCAP ${ }^{\circledR}$.

\subsection{Enzyme-linked immunosorbent assay (ELISA) for CN component specific IgE levels}

The as1-, $\beta$-, and $x$-CN-specific IgE levels were evaluated by indirect ELISA. ${ }^{4}$ The relative immunoglobulin levels (\%) for each sample were calculated based on the reference serum with high as1-CN-specific immunoglobulin levels. These measured values were used in this study.

\subsection{Competitive ELISA}

The inhibition ratios of $\beta$ - and $\chi$-CN against $\alpha$ s1-CN-specific IgE were evaluated by competitive ELISA. The 96-well microtiter plates (Nunc-ImmunoTM Plate II, Thermo Fisher Scientific) were coated with as1-CN (5 $\mu \mathrm{g} / \mathrm{mL}$ in PBS) and incubated overnight at 4 . After washing the plates with PBS containing $0.05 \%$ Tween 20 (PBS-T), the plates were blocked with PFBB containing 0.05\% Tween 20 (PFBB-T) for $2 \mathrm{~h}$ at 4 . Patient sera $(\mathrm{n}=39)$ were diluted $1: 5$ in PFBB-T and pre-incubated with inhibitor (as1-, $\beta$-, and $x$-CN diluted in PBS at the final concentrations of $0.001-100 \mu \mathrm{g} / \mathrm{mL}$ ). The pre-incubated sera were added to each well and incubated overnight at 4 . After washing each well with PBS-T, bound antibodies were detected with AP-conjugated goat anti-human IgE (diluted 1:1,000 in PFBB-T) for $1.5 \mathrm{~h}$ at 37 . After washing each well with PBS-T and PBS, PNPP substrate (Thermo Fisher Scientific) was added. The reaction was stopped by the addition of $2 \mathrm{~N} \mathrm{NaOH}$ and the absorbance was measured at $405 \mathrm{~nm}$. The inhibition ratio (\%) was calculated using the following formula:

Inhibition ratio $(\%)=100-($ Inhibitor $-\mathrm{NC}) /(\mathrm{PC}-\mathrm{NC}) \times 100$

Inhibitor: absorbance of inhibitor solution; NC: Negative control, absorbance of PFBB-T; PC: Positive control, absorbance of serum without inhibitor

\subsection{Sodium dodecyl sulfate- polyacrylamide gel electrophoresis (SDS-PAGE) and immunoblot- ting}

The purity of the isolated $\alpha$ s1-CN and contamination of isolated $\beta$-CN was confirmed by SDS-PAGE and immunoblotting. The gel (15\% polyacrylamide) was stained with Coomassie Brilliant Blue R-250 (FUJIFILM Wako Pure Chemical Corporation, Osaka, Japan). For immunoblotting, proteins were transferred to polyvinylidene difluoride membranes. After blocking ( $1 \% \mathrm{w} / \mathrm{v}$ bovine serum albumin in tris-buffered saline), as1-CN was detected by anti- $\alpha$-casein mouse monoclonal antibody and peroxidase-conjugated anti-mouse IgG.

\subsection{Bioinformatics analysis}


To quantify the similarity between as1-CN and the other CN components, the Property Distance $(P D$ ) value was calculated using the Property-Based Peptide Similarity Index PD for Two Sequences tool (http://Fermi.utmb.edu/ SDAP/sdap_pdi.html). ${ }^{8}$ In brief, the sequential peptides consisting of ten aminoacids, overlapping by seven (three-offset), in the primary sequence of as1-CN were applied to the amino-acid sequences of $\beta$ - and $\chi$-CN. Peptides with $P D$ values lower than 10 indicated significant physicochemical similarities, whereas much higher $P D$ values implied unrelated peptides. In addition, to identify the putative cross-reactive peptides, $\alpha$ s1-CN-specific IgE epitopes reported by the previous studies were also applied to the amino acid sequences of $\alpha$ s2-, $\beta-$, and $x$-CN.

\subsection{Statistical analysis}

Correlations among $\alpha$ s1-, $\beta$-, and $\chi$-CN-specific IgE were analyzed by Pearson's correlation analysis. Differences among the inhibition rate of CN components were analyzed by one-way analysis of variance (ANOVA). Calculations were performed with EZR software produced by the Jichi Medical University Saitama Medical Center (Saitama, Japan). ${ }^{9}$ Data are expressed as the mean \pm standard deviation (SD). Differences with $P$ values of less than 0.05 were considered statistically significant.

\subsection{Ethics statement}

This study was conducted in accordance with the Declaration of Helsinki. Written informed consent was obtained from the parents; patient's assent was also obtained at the time of oral food challenge test, including the use of stocked sera for research purposes. This current study, which used the stocked sera, was again approved by the institutional ethical committee at Aichi Children's Health and Medical Center (\#201669) and at Nagoya University of Arts and Sciences (\#200).

\section{RESULTS}

\subsection{Correlations between casein components-specific immunoglobulins}

Correlations between immunoglobulin levels of each CN component are shown in Figure 1. The as1-CNspecific IgE levels exhibited a strong positive correlation with the $\beta$-CN-specific $\operatorname{IgE}(\mathrm{r}=0.945, P<0.001$, Figure 1A). $x$-CN-specific IgE levels showed relatively lower correlations between $\alpha$ s1-CN-specific IgE levels $(\mathrm{r}=0.57, P<0.001$; Figure $1 \mathrm{~B})$ and $\beta$-CN-specific IgE levels $(\mathrm{r}=0.616, P<0.001$; Figure $1 \mathrm{C})$. These results suggested that the patients with $\mathrm{CM}$ allergy were sensitized to $\alpha$ s1- and $\beta$-CN at the same time, and/or a cross-reactive epitope exists between these two CNs.

Moreover, as1-, $\beta$-, and $x$-CN-specific IgG4 and IgA levels were also investigated (Figure S1). The specific IgG4 levels showed the same tendency with IgE. However, IgA levels showed that not only $\beta-\mathrm{CN}$, but also $x$-CN had a strong correlation with as1-CN.

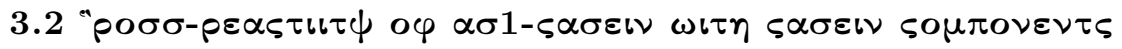

To confirm whether a cross-reactive epitope exists between the as1- and $\beta$-CN components, we performed a competitive ELISA of $\beta$-CN against $\alpha$ s1-CN-specific IgE (Figure 2). Complete inhibition was observed at high concentrations of $\beta-\mathrm{CN}(10-100 \mu \mathrm{g} / \mathrm{mL}$, Figure $2 \mathrm{~A})$. The $\mathrm{IC}_{50}$ of the $\beta-\mathrm{CN}(0.160 \mu \mathrm{g} / \mathrm{mL})$ was about 10 times higher than that of $\alpha \mathrm{s} 1-\mathrm{CN}$ itself $(0.0139 \mu \mathrm{g} / \mathrm{mL})$, suggesting differences in the number or the affinity of IgE epitopes. On the other hand, the $\mathrm{IC}_{50}$ of $\chi-\mathrm{CN}(13.6 \mu \mathrm{g} / \mathrm{mL})$ was about 1,000 times higher than that of $\alpha$ s1-CN, and the maximum inhibition rate remained $54.5 \%$ at the highest concentration (Figure $2 \mathrm{~B}$ ). These results suggested the possibility that a cross-reactive epitope exists between as1- and $\beta$-CN.

To exclude the possibility that as1-CN was contaminated with the purified $\beta$-CN, we performed SDS-PAGE and immunoblotting using $\alpha$-casein-specific monoclonal antibody. Even in the ten times more protein supplementation of $\beta$-CN by SDS-PAGE, no bands detected by the as1-CN-specific immunoblotting (Figure S2). Therefore, the possibility of protein contamination was unlikely.

\subsection{In silico analysis of the partial similarity of amino acid sequence}


Using the Property-Based Peptide Similarity Index PD for Two Sequences tool, we detected 24 peptide sets between $\alpha$ s1- and $\beta-\mathrm{CN}$ and 14 sets between $\alpha$ s1- and $x-\mathrm{CN}$ that showed $P D$ values lower than 10 (Table 1 ). The lowest $P D$ value, 5.30, was detected between amino acid sequences of $\alpha$ s1-CN (E61-E70) and $\beta$-CN (I12-E21). On the other hand, the lowest $P D$ value between $\alpha$ s1-CN and $\chi$-CN was 8.47. Furthermore, we found 38 peptide sets with $P D$ values lower than 10 between $\alpha$ s1- and $\alpha$ s2-CN, 34 sets between $\alpha$ s2- and $\beta$-CN, 19 sets between $\alpha$ s- and $x$-CN, and 18 sets between $\beta$ - and $x$-CN (Table S2).

In the above results, the domains where $P D$ values were low existed between $\alpha$ s- and $\beta$-CN. The peptide sets with the lowest $P D$ values between each $\mathrm{CN}$ component are shown in Table 2. As a result, $\alpha$ s1-CN had a particularly low $P D$ value against $\beta$ - and $x-\mathrm{CN}$ and the amino acid compositions in these peptides were identical in $7 / 10$ and $3 / 10$ residues, respectively. On the other hand, identical amino acid compositions of as2-CN against as1- and $\beta$-CN were 6/10 and 7/10 residues, respectively. Of note, these peptides included the particular "SSSEE" amino acid sequence.

\subsection{In silico analysis of the known IgE epitopes of CN components}

To identify the putative cross-reactive epitope peptides in as1-CN, we also applied in silico analysis on the previously reported $\operatorname{IgE}$ epitopes of $\alpha \mathrm{s} 1-\mathrm{CN}^{10-13}$ against the amino acid sequences of $\alpha \mathrm{s} 2-, \beta-$, and $x$ CN. Unexpectedly, we could only find six peptide sets that showed $P D$ values lower than 10 (Table 3). Furthermore, the lowest $P D$ value of 7.16 was detected in as2-CN.

We arranged the amino acid sequences of casein components and filled the reported IgE epitopes in order to visualize the location (Figure 3). ${ }^{10-15}$ The four "SSSEE" motifs, known as the core motif of casein phosphopeptide (CPP), are shown in bold characters, but the reported IgE epitopes were scarcely located on the "SSSEE" motif except one located at the N-terminus of as2-CN.

\section{DISCUSSION}

The purpose of this study was to investigate the cross-reactivity among CN components. The data clearly indicated that IgE (also IgG4 and IgA) specific to $\alpha$ s1- and $\beta$-CN were strongly associated, and the presence of strong cross-reactivity was confirmed using competitive ELISA. We also found lower cross-reactivity between as1-CN and $x$-CN. Unfortunately, we could not examine $\alpha$ s2-CN due to purification difficulties.

Bernard et al. reported that the sum of the IgE levels to the different $\mathrm{CN}$ components was higher than the IgE levels to whole $\mathrm{CN}$, suggesting the presence of cross-reactive epitopes between the CN components. ${ }^{16}$ Generally, cross-reactivity is expected between proteins with high sequence homology. ${ }^{17}$ However, an article which compiled cross-reactivities between non-homologous allergens was recently reported. ${ }^{18}$ With regard to $\mathrm{CN}$, the homology of full-length amino acid sequences between CN components is extremely low (4-7\%). To assess this theoretical gap in knowledge, we employed in silico analysis to find the common peptide sequences in each CN component and found highly homologous amino acid sequences in $\alpha$ s1-, $\alpha$ s2-, and $\beta-\mathrm{CN}$. The sequences commonly included the unique "SSSEE" motif, known as the basic structure of CPP.

During $\mathrm{CN}$ digestion, $x$-CN, which covers the surface of $\mathrm{CN}$ micelles, is broken down and subsequently the other $\mathrm{CNs}$ too. ${ }^{19} \mathrm{CN}$ is well known as a protein with high digestibility. ${ }^{20}$ However, the fact that $\mathrm{CN}$ is a major allergen suggests that the major IgE epitope should be placed in the digestion-resistant structure. ${ }^{21}$ $\mathrm{CPP}$, which results from the digestion of $\mathrm{CN}$, has been identified as a trypsin-resistant peptide due to the highly phosphorylated serine residues in and around the core structure of "SSSEE". CPP plays a key role in the effective absorption of calcium due to the formation of micellar calcium phosphate at the phosphorylated serine residues. Based on this nutritional function, CPP is widely used medically to prevent osteoporosis and in dental care to ameliorate the calcium deposition on teeth. Remarkably, dental treatments using small amounts of CPP paste have the potential to be allergenic and cause anaphylaxis. This clinical fact suggests that CPP presents strong allergenic activity. CPP derived from $\alpha$ s- and $\beta$-CN. CPP identified in $\alpha$ s1-CN (D43-K79), as2-CN (K1-K32 and N46-K70) and $\beta$-CN (R1-K28) (Figure 3). ${ }^{22,23}$

In this study, we did not find strong cross-reactivity and detect a highly homologous amino acid sequence between as1-CN and $x$-CN. The CPP motif does not exist in $x$-CN, and the C-terminal half of $x$-CN consists of 
a unique water-soluble glycoprotein designated as a glycomacropeptide. ${ }^{24}$ Epitope-mapping of $x$-CN reveals many IgE binding sites, which might have individual allergenic properties with low cross-activity between $\alpha$ - and $\beta$-CNs. Epitope-mapping of whole CN components has been extensively performed worldwide, using overlapping peptides and peptide microarray techniques. ${ }^{10-13}$ Nonetheless, the reported IgE-epitopes are different with each other, and no consensus of the dominant IgE epitope has been established. Furthermore, prior to this study, cross-reactive epitopes among CN components have not been reported.

Recently, IgE- and IgG4-epitope mapping was carried out to develop a prediction tool for the prognosis of $\mathrm{CM}$ allergy. ${ }^{25-} 29$ However, in these reports, there were few epitope sequences located in the CPP regions and no common epitopes were found in the CPP regions on the different CN components. We supposed the reason that IgE binding ability to synthetic peptide is low compared with natural $\mathrm{CN}$ is because serine residues were not phosphorylated in the synthetic peptides.

Generally, IgE-binding epitopes have hydrophilic properties and are present on the surface of a granular protein molecule. Phosphorylated serine residues comprise the water-soluble part, and low phosphorylated residues comprise the water-insoluble part of the $\mathrm{CN}$ molecule. Otani et al. reported that the IgG binding ability to dephosphorylated $\alpha$ s1-CN was lower than that to native $\alpha$ s1-CN. ${ }^{30}$ Furthermore, while $O$-phosphoL-serine inhibited IgG binding, L-serine scarcely inhibited binding, thereby indicating the importance of the phosphorylated serine residue in the epitope. Therefore, phosphorylated serine residues in the CPP motifs might have the critical role in the formation of the dominant and cross-reactive IgE epitopes in the native CN components.

A major limitation of this study is that we suggest the presence of cross-reactive epitopes between as1-CN and $\beta$-CN based on bioinformatics finding of the presence of highly homologous amino acid sequences in the $\mathrm{CPP}$ regions; however, we need to combine these findings with direct experimental data. Therefore, future experiments are expected to define the IgE-binding capacity of highly purified CPPs from natural CN and evaluate the effect of dephosphorylation. If this agenda were achieved, this would suggest the following: (i) CPP can induce milk allergy, (ii) possibility of cross-reaction caused by the similarity of CPP motifs on the different $\mathrm{CN}$ components, (iii) change in IgE binding ability by dephosphorylating the side chain of an amino acid, and (iv) partial negation of epitope analysis using the conventional overlapping peptides.

In conclusion, despite the homology among CN components being extremely low, as1-CN shows crossreactivity with $\beta$-CN. The sequence of "SSSEE", which is a basic structure of CPP, may have a potential of being the cross-reactive epitope.

\section{Acknowledgements}

The authors are grateful to the former staff of Aichi Children's Health and Medical Center for collecting the serum samples over a span of many years. The authors thank Editage for English language editing (www.editage.com).

\section{Author Contributions}

MN, TM, KI, and HI designed the study and drafted the manuscript. TM, KT, and KI collected and sorted the serum samples. MN, CY, and HI performed ELISA and inhibition assay. MN performed in silicoanalysis and statistical analysis. TM and KI reviewed the manuscript. All authors approved the final submitted manuscript.

\section{Impact Statement}

The homology among casein (CN) components is extremely low, but as1-CN and $\beta$-CN show cross-reactivity. Based on these results, there is a need for detailed examination of the allergenicity of casein phosphopeptides, which includes common sequences of $\alpha$ s1-CN and $\beta-\mathrm{CN}$.

\section{REFERENCES}


1. Ito K, Futamura M, Movérare R, et al. The usefulness of casein-specific IgE and IgG4 antibodies in cow's milk allergic children. Clin Mol Allergy . 2012;10:1.

2. Ono T. Applied aspects derived from the relationship between calcium and milk proteins. Jpn. Milk Sci (in Japanese) . 2005;54:53-62.

3. Farrell HM Jr, Jimenez-Flores R, Bleck GT, et al. Nomenclature of the proteins of cows' milk-sixth revision. J Dairy Sci . 2004;87:1641-1674.

4. Naito M, Matsui T, Tagami K, et al. Changes in casein component-specific antibody levels during oral immunotherapy for milk allergy. Pediatr Allergy Immunol . 2020;31:421-424.

5. Candreva ÁM, Smaldini PL, Curciarello R, Fossati CA, Docena GH, Petruccelli S. The major soybean allergen Gly $\mathrm{m}$ Bd $28 \mathrm{~K}$ induces hypersensitivity reactions in mice sensitized to cow's milk proteins. $J$ Agric Food Chem . 2016;64:1590-1599.

6. Candreva AM, Smaldini PL, Curciarello R, et al. Cross-reactivity between the soybean protein p34 and bovine caseins. Allergy Asthma Immunol Res . 2015;7:60-68.

7. Matsui T, Naito M, Tagami K, et al. Changes in passively-sensitized basophil activation to $\alpha \mathrm{S} 1$-casein after oral immunotherapy.Immun Inflamm Dis . 2020;8:188-197.

8. Ivanciuc $\mathrm{O}$, Midoro-Horiuti $\mathrm{T}$, Schein $\mathrm{CH}$, et al. The property distance index PD predicts peptides that cross-react with IgE antibodies. Mol Immunol . 2009;46:873-883.

9. Kanda Y. Investigation of the freely available easy-to-use software 'EZR' for medical statistics. Bone Marrow Transplant . 2013;48:452-458.

10. Chatchatee P, Järvinen KM, Bardina L, Beyer K, Sampson HA. Identification of IgE- and IgG-binding epitopes on alpha(s1)-casein: differences in patients with persistent and transient cow's milk allergy. $J$ Allergy Clin Immunol . 2001;107:379-383.

11. Spuergin P, Mueller H, Walter M, Schiltz E, Forster J. Allergenic epitopes of bovine alpha S1-casein recognized by human IgE and IgG.Allergy . 1996;51:306-312.

12. Cong Y, Yi H, Qing Y, Li L. Identification of the critical amino acid residues of immunoglobulin $\mathrm{E}$ and immunoglobulin G epitopes on as1-casein by alanine scanning analysis. J Dairy Sci . 2013;96:68706876 .

13. Cerecedo I, Zamora J, Shreffler WG, et al. Mapping of the IgE and IgG4 sequential epitopes of milk allergens with a peptide microarray-based immunoassay. J Allergy Clin Immunol . 2008;122:589-594.

14. Chatchatee P, Järvinen KM, Bardina L, Vila L, Beyer K, Sampson HA. Identification of IgE and IgG binding epitopes on beta- and kappa-casein in cow's milk allergic patients. Clin Exp Allergy . 2001;31:1256-1262.

15. Busse PJ, Järvinen KM, Vila L, Beyer K, Sampson HA. Identification of sequential IgE-binding epitopes on bovine alpha(s2)-casein in cow's milk allergic patients. Int Arch Allergy Immunol . 2002;129:93-96.

16. Bernard H, Créminon C, Yvon M, Wal JM. Specificity of the human IgE response to the different purified caseins in allergy to cow's milk proteins. Int Arch Allergy Immunol . 1998;115:235-244.

17. Restani P, Ballabio C, Di Lorenzo C, Tripodi S, Fiocchi A. Molecular aspects of milk allergens and their role in clinical events. Anal Bioanal Chem . 2009;395:47-56.

18. Bublin M, Breiteneder H. Cross-reactivities of non-homologous allergens. Allergy . 2020;75:1019-1022.

19. Hashida S, Ishikawa E, Nakamichi N, et al. Concentration of egg white lysozyme in the serum of healthy subjects after oral administration. Clin Exp Pharmacol Physiol . 2002;29:79-83.

20. Wal JM. Structure and function of milk allergens. Allergy . 2001;56 Suppl 67:35-38.

21. Sakurai N, Nishio S, Akiyama Y, et al. Apical-to-basolateral transepithelial transport of cow's milk caseins by intestinal Caco-2 cell monolayers: MS-based quantitation of cellularly degraded $\alpha$ - and $\beta$ casein fragments. $J$ Biochem 2018;164:113-125.

22. Hirayama M, Toyota K, Yamaguchi G, Hidaka H, Naito H. HPLC analysis of commercial casein phosphopeptides (CPP). Biosci Biotechnol Biochem . 1992;56:1126-1127.

23. Juillerat MA, Baechler R, Berrocal R, Chanton S, Scherz JC, Jost R. Tryptic phosphopeptides from whole casein. I. Preparation and analysis by fast protein liquid chromatography. J Dairy Res . 1989;56:603-611.

24. Rutherfurd KJ, Gill HS. Peptides affecting coagulation. Br J Nutr . 2000;84 Suppl 1:S99-102. 
25. Wang J, Lin J, Bardina L, et al. Correlation of $\operatorname{IgE} / \operatorname{IgG} 4$ milk epitopes and affinity of milk-specific $\operatorname{IgE}$ antibodies with different phenotypes of clinical milk allergy. J Allergy Clin Immunol . 2010;125:695702, 702.e1-702.e6.

26. Savilahti EM, Rantanen V, Lin JS, et al. Early recovery from cow's milk allergy is associated with decreasing IgE and increasing IgG4 binding to cow's milk epitopes. J Allergy Clin Immunol . 2010;125:1315-1321.e9.

27. Järvinen KM, Beyer K, Vila L, Chatchatee P, Busse PJ, Sampson HA. B-cell epitopes as a screening instrument for persistent cow's milk allergy. J Allergy Clin Immunol . 2002;110:293-297.

28. Caubet JC, Lin J, Ahrens B, et al. Natural tolerance development in cow's milk allergic children: $\operatorname{IgE}$ and IgG4 epitope binding. Allergy . 2017;72:1677-1685.

29. Savilahti EM, Kuitunen M, Valori M, et al. Use of IgE and IgG4 epitope binding to predict the outcome of oral immunotherapy in cow's milk allergy. Pediatr Allergy Immunol . 2014;25:227-235.

30. Otani H, Hori H, Hosono A. Antigenic reactivity of dephosphorylated as1-casein, phosphopeptide from $\beta$-casein and $O$-phospho-L-serine towards the antibody to native as1-casein. Agric Biol Chem . 1987;51:2049-2054.

Table 1. Putative peptides similarity between $\alpha$ s1-casein and $\beta$-casein and between $\alpha$ s1-casein and $x$-casein (in silico analy: Amino acid

as1-Casein

$7-16$

$7-16$

$22-31$

$28-37$

$31-40$

$34-43$

$40-49$

$46-55$

$46-55$

$46-55$

$49-58$

$55-64$

$61-70$

$61-70$

$64-73$

$79-88$

$94-103$

$97-106$

$109-118$

$112-121$

$115-124$

$118-127$

$124-133$

$139-148$

performed by Structural Database of Allergenic Proteins (SDAP, http://Fermi.utmb.edu/SDAP/). The each peptide has $P$.

Table 2. The combination of partial amino acid sequence which has lowest $P D$ value between each casein

Table 2. The

as1-Casein

as2-Casein

$\alpha \mathrm{s} 1$

$\alpha \mathrm{s} 2$

$P D$ 
as2-Casein

$\beta$-Casein

The matched amino acids were shown by under line.

The matched :

Table 3. Identification of putative cross-reactive peptides of $\alpha$ s1-casein-specific IgE epitopes

Ref.

10

11

12

13

performed by Structural Database of Allergenic Proteins (SDAP, http://Fermi.utmb.edu/SDAP/). The $\alpha$ s1-CN-sIgE epitop

\section{Figure Legends}

Figure 1

Specific-IgE levels (\%) to $\alpha$ s1- and $\beta$-casein (A), to $\alpha$ s1- and $x$-casein (B), and $\beta$ - and $x$-casein (C) were plotted. Correlation analysis was performed by linear regression.

\section{Figure 2}

The cross-reactivity of $\alpha$ s1-casein was determined by competitive ELISA (A). The $\alpha$ s1-casein (circle) was used as the control, and $\beta$-casein (triangle) and $x$-casein (square) were used as inhibitors $(\mathrm{n}=39)$. The $\mathrm{IC}_{50}$ is the concentration of inhibitor that causes a $50 \%$ inhibition of antibody binding (B).

\section{Figure 3}

Schematic representation of each casein's amino acid sequence and determined peptides. The IgE epitopes are shown by the dotted line (ref. 10), broken line (ref. 11), solid line (ref. 12), dotted line and triangle (ref. 13), broken line and triangle (ref. 14), and solid line and triangle (ref. 15). Casein phosphopeptide (CPP), which is identified by trypsin digestion, is shown by the shaded region. Amino acid sequence, SSSEE, is shown in bold.

\section{Hosted file}


Figures_and_Tables_200623.pdf available at https://authorea.com/users/336797/articles/462492evaluation-of-cross-reactivity-between-casein-components-using-inhibition-assay-and-insilico-analysis

(A)

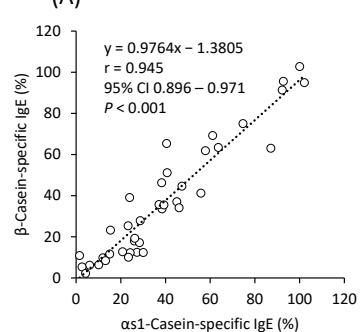

(B)

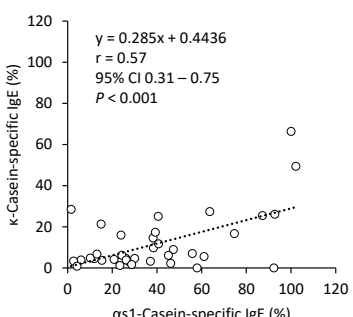

(C)

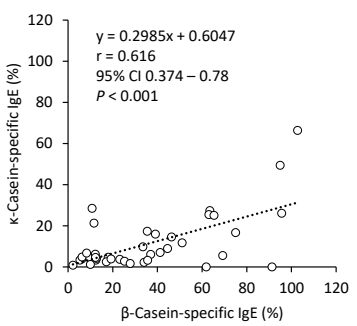

